\title{
LA «SORORITÉ » À L'ÉPREUVE : PRATIQUER L'INTERNATIONALISME FÉMINISTE AU LENDEMAIN DE LA GUERRE FROIDE
} Ioana Cîrstocea

Presses de Sciences Po (P.F.N.S.P.) | « Critique internationale »

2015/1 N66 | pages 85 à 101

ISSN 1290-7839

ISBN 9782724633962

Article disponible en ligne à l'adresse :

http://www.cairn.info/revue-critique-internationale-2015-1-page-85.htm

\section{Pour citer cet article :}

Ioana Cîrstocea, « La « sororité » à l'épreuve : pratiquer l'internationalisme féministe au lendemain de la guerre froide », Critique internationale 2015/1 (N 66), p. 85-101. DOI 10.3917/crii.066.0085

Distribution électronique Cairn.info pour Presses de Sciences Po (P.F.N.S.P.).

(C) Presses de Sciences Po (P.F.N.S.P.). Tous droits réservés pour tous pays.

La reproduction ou représentation de cet article, notamment par photocopie, n'est autorisée que dans les limites des conditions générales d'utilisation du site ou, le cas échéant, des conditions générales de la licence souscrite par votre établissement. Toute autre reproduction ou représentation, en tout ou partie, sous quelque forme et de quelque manière que ce soit, est interdite sauf accord préalable et écrit de l'éditeur, en dehors des cas prévus par la législation en vigueur en France. Il est précisé que son stockage dans une base de données est également interdit. 


\section{La « sororité »} à l'épreuve : pratiquer I'internationalisme féministe au lendemain de la guerre froide

par loana Cîrstocea

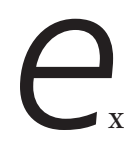

xemple par excellence de la « transnationalisation $\gg^{1}$ des solidarités politiques et des pratiques militantes, le féminisme est l'un des thèmes revisités à la faveur du « tournant de l'après-guerre froide » qui met en avant la porosité des frontières et la multiplicité des circulations entre les deux « blocs $»^{2}$. Des recherches ont été ainsi consacrées aux contacts établis dans le cadre de divers événements institutionnels entre des groupes de femmes vivant de chaque côté du rideau de fer, alors que, faute de sources pouvant les documenter, les échanges

1. Pascale Dufour et al. (eds), Solidarities beyond Borders: Transnationalizing Women's Movements, VancouverToronto, UBC Press, 2010 ; Johanna Siméant, « La transnationalisation de l'action collective », dans Éric Agrikoliansky, Olivier Fillieule, Isabelle Sommier (dir.), Penser les mouvements sociaux. Conflits sociaux et contestations dans les sociétés contemporaines, Paris, La Découverte, 2010, p. 123-143.

2. Sari Chari, Katherine Verdery, «Thinking between the Posts: Post-colonialism, Post-socialism, and Ethnography after the Cold War », Comparative Studies in Society and History, 51 (1), 2009, p. 6-34 ; Justine Faure, Sandrine Kott (dir.), « Le Bloc de l'Est en question », Vingtième Siècle. Revue d'bistoire, dossier thématique, 109, 2011. 
moins formalisés sont restés peu étudiés ${ }^{3}$. Nous ne prétendons pas combler cette lacune et notre démonstration porte d'ailleurs sur des interactions situées audelà des limites chronologiques de la guerre froide. Toutefois, en nous centrant sur la mise en place en 1990 d'un réseau féministe transatlantique, le Network of East-West Women (NEWW), nous montrerons la persistance du cadrage idéologique « Est vs Ouest». En effet, tout en renouvelant l'internationalisme féministe, les protagonistes des interactions que nous étudions interprètent leur réalité en termes de décalage et de différence, voire de conflit. Et au moment même où la configuration géopolitique bipolaire est en train de s'effacer, la catégorie « femmes est-européennes » se construit en support de mobilisation collective et sert à politiser une identité féministe non hégémonique. Suivant un programme de sociologie politique sensible, d'une part, à l'inscription temporelle et à la subjectivité des engagements militants ${ }^{4}$, d'autre part, au caractère hybride et à la multiplicité des formes de mobilisation féministe ${ }^{5}$, nous saisirons les trajectoires de ces militantes à travers les dimensions subjectives de leur identification à des mouvements localisés de libération des femmes, au féminisme global et aux processus de libéralisation des pays ex-socialistes.

Précisons avant tout que, dans les pays ex-socialistes, le féminisme des années 1990 s'inscrit dans des logiques à la fois académiques (programmes universitaires de women's/gender studies) et associatives (organisations de promotion des droits des femmes soutenues par des programmes de démocratisation) et qu'il révèle des processus d'innovation intellectuelle et institutionnelle impulsés par l'affirmation $\mathrm{du}$ « genre » comme norme démocratique à prétention universelle et comme outil de gouvernance globale ${ }^{6}$. Marquée par plusieurs événements organisés sous l'égide

3. Raluca Popa, « Translating Equality between Women and Men across Cold War Divides: Women Activists from Hungary and Romania and the Creation of International Women's Year », dans Jill Massino, Shana Penn (eds), Gender Politics and Everydaylife in State Socialist Eastern and Central Europe, New York, Palgrave Macmillan, 2009, p. 59-74 ; Francisca De Haan, « Continuing Cold War Paradigms in the Western Historiography of Transnational Women's Organizations: The Case of the Women's International Democratic Federation », Women's History Review, 19 (4), 2010, p. 547-573 ; Karen Garner, Shaping a Global Women's Agenda: Women's NGOs and Global Governance, 1925-1985, Manchester, New York, Manchester University Press, 2010 ; Kristen Ghodsee, « Revisiting the International Decade for Women: Brief Reflections on Competing Definitions of Feminism and Cold War Politics from the American Perspective », Women's Studies International Forum, 33, 2010, p. 3-12 ; Melanie Ilic, « Soviet Women, Cultural Exchange and the Women's International Democratic Federation », dans Sari Autio-Sarasmo, Katalin Miklossy (eds), Reassessing Cold War Europe, Londres, New York, Routledge, 2011, p. 157-174 ; Chiara Bonfiglioli «Women's Political and Social Activism in the Early Cold War Era: The Case of Yugoslavia », Aspasia, The International Yearbook of Central, Eastern, and Southeastern European Women's and Gender History, 8, 2014, p 1-25 ; Jean H. Quataert, Benita Roth (eds), « Human Rights, Global Conferences, and the Making of Postwar Transnational Feminisms », Fournal of Women's History, dossier thématique, 24 (4), 2012. 4. É. Agrikoliansky, O. Fillieule, I. Sommier (dir.), Penser les mouvements sociaux, op. cit. ; Olivier Fillieule, Lilian Mathieu, Cécile Péchu (dir.), Dictionnaire des mouvements sociaux, Paris, Presses de Sciences Po, 2009.

5. Laure Bereni, Anne Revillard (dir.), « Les femmes contestent. Genre, féminisme, et mobilisations collectives », Sociétés contemporaines, dossier thématique, 85 (1), 2012.

6. Monique Selim, « L'émancipation des femmes au XXI ${ }^{e}$ siècle, une pierre dans la gouvernance du capitalisme globalisé », dans Françoise Picq, Martine Storti (dir.), Le féminisme à l'épreuve des mutations géopolitiques. Congrès international féministe, Paris décembre 2010, Paris, Éditions iXe, 2012, p. 183-197; M. Selim, « La face cachée des femmes outils », L'Homme et la Société, 176-177 (2-3), 2010, p. 253-266; Delphine Lacombe et al. (dir.), « Le genre globalisé. Cadres d'action et mobilisations en débat », Cultures et Conflits, dossier thématique, 83, 2011. 
des Nations unies, la décennie 1990 est celle de l'institutionnalisation plénière des « droits des femmes en tant que droits humains » et du gender mainstreaming comme principe d'action publique. Différentes recherches ont mis en lumière l'importance des dynamiques et des ressources internationales dans les processus de légitimation de ces thématiques dans les pays est-européens, où elles ont été envisagées comme moteur de changement démocratique et ont fait l'objet d'un véritable apprentissage transnational' ${ }^{7}$. Ayant travaillé sur la sociologie de la promotion internationale du genre, nous avons étudié l'aide accordée par la fondation Soros (Open Society Institute, OSI) à la formation intellectuelle des pionnières féministes est-européennes, dans le cadre de programmes d'études ou d'offres de soutien aux associations militant pour les droits des femmes. ${ }^{8}$. Menée en étroite relation avec l'organisation philanthropique, l'action du NEWW s'est révélée cruciale pour les échanges internationaux autour du genre en Europe postsoviétique. Nous avons choisi de centrer ici notre argumentation sur la mise en place, la composition et la stratégie du réseau, voire sur les dispositions et les pratiques de ses fondatrices, afin de discuter la construction symbolique de l'opposition « Est-Ouest » et le projet de son dépassement par une politique féministe « transversale »?.

Notre démonstration s'appuie sur une enquête menée en automne 2010 aux ÉtatsUnis, au cours de laquelle nous avons conduit une dizaine d'entretiens auprès de membres et de sympathisantes du NEWW et dépouillé les archives non déposées

7. K. Ghodsee, « Feminism-by-Design: Emerging Capitalisms, Cultural Feminism, and Women's NGOs in Post-socialist Eastern Europe », Signs. Fournal of Women in Culture and Society, 29 (3), 2004, p. 727-753 ; Alexandra Hrycak, «Foundation Feminism and the Articulation of Hybrid Feminisms in Post-Socialist Ukraine », East-European Politics and Societies, 20, 2006, p. 69-100 ; Julie Hemment, Empowering Women in Russia. Activism, Aid and NGOS, Bloomington, Indiana University Press, 2007 ; Susan Zimmermann, « The Institutionalization of Women's and Gender Studies in Higher Education in Central and Eastern Europe and the Former Soviet Union: Asymmetric Politics and the Regional-Transnational Configuration », East Central Europe/ECE, 34, 2007, p. 131-160 ; Eva Fodor, Eszter Varsa, « At the Crossroads of East and West: Gender Studies in Hungary », dans Christine Ellen Bose, Kim Minjeong (eds), Global Perspectives on Gender Research: Transnational Perspectives, New York, Londres, Routledge, 2009, p. 290-307 ; Katerina Pishchikova, Promoting Democracy in Post-communist Ukraine: The Contradictory Outcomes of US Aid to Women's NGOs, Boulder, Colorado, First Forum Press, 2011 ; Krasimira Daskalova (ed.), « The Birth of a Field: Women's and Gender Studies in Central, Eastern and South-Eastern Europe, I », Aspasia. The International Yearbook of Central, Eastern and South East European Women's and Gender History, 4, 2010, p. 155-205 ; K. Daskalova (ed.), « The Birth of a Field: Women's and Gender Studies in Central, Eastern and South-Eastern Europe, II », Aspasia. The International Yearbook of Central, Eastern and South East European Women's and Gender History, 5, 2011, p. 128-203.

8. Ioana Cîrstocea, «Éléments pour une sociologie des études féministes en Europe centrale et orientale », International Review of Sociology/Revue internationale de sociologie, 20 (2), 2010, p. 321-346 ; I. Cîrstocea, « The Transnational Feminism in the Making: The Case of Post-Communist Eastern Europe », dans Claudette Fillard, Françoise Orazi (eds), Exchanges and Correspondence: The Construction of Feminism, Cambridge, Cambridge Scholars Publishing, 2010, p. 64-83 ; I. Cîrstocea, « Lieux et formes de transnationalisation du féminisme : le cas de l'Europe de l'Est depuis $1990 »$, dans Marie-Pierre Arrizabalaga et al. (dir.), Femmes sans frontières : stratégies transnationales féminines face à la mondialisation (XVII ${ }^{e}-X X^{e}$ siècles), Berne, Peter Lang, 2011, p. 385-402 ; I. Cîrstocea, «Après la chute du Mur de Berlin : voies et formats du féminisme transnational », dans F. Picq, M. Storti (dir.), Le féminisme à l'épreuve des mutations géopolitiques. Congrès international féministe, Paris décembre 2010, op. cit., p. 160-169.

9. Nira Yuval Davis, Gender and Nation, Londres, New York, Sage, 1997. 
du réseau se trouvant en possession de la présidente fondatrice, Ann Snitow ${ }^{10}$. Des éléments d'information en provenance des archives de l'OSI à Budapest et différents types de sources secondaires (études publiées, informations accessibles en ligne) sont également mobilisés de façon ponctuelle. Nous présenterons tout d'abord la genèse du réseau, ses ressources et ses activités durant ses premières années d'existence, puis discuterons deux éléments centraux de son idéologie : la « sororité $»^{11}$ et la « division Est-Ouest $»^{12}$. Nous montrerons ainsi que, tout en déployant des activités transatlantiques et en investissant de nouveaux registres d'action politique, les militantes du NEWW ont contribué pendant la première décennie postcommuniste à reproduire le clivage idéologique de la guerre froide, qui était, en quelque sorte, la raison d'être du réseau.

\section{"Faire naître un dialogue féministe à travers les frontières " $^{13}$}

Tout a commencé en 1990, à New York, lors de la Socialist Scholars' Conference, événement traditionnel de la gauche intellectuelle américaine depuis le début des années 1960. La communication de l'écrivaine et sociologue croate Slavenka Drakulić, centrée sur les difficultés propres à la condition des femmes pendant les régimes socialistes ${ }^{14}$ et formulée dans un esprit polémique, interpella quelques universitaires féministes américaines présentes à la manifestation. Celles-ci décidèrent par la suite d'organiser une rencontre internationale afin d'en apprendre davantage sur les expériences et les préoccupations des femmes dans les pays socialistes. L'événement fut préparé par un petit groupe de militantes « historiques » du mouvement états-unien pour la libération des femmes, qui mobilisèrent efficacement leur expérience et leur savoir-faire en matière de communication, d'organisation, de diffusion d'informations et de collecte de fonds. Présentée par ses animatrices comme une première historique, cette rencontre des femmes

10. Nous remercions vivement Ann Snitow pour le soutien qu'elle a apporté à notre travail. Nous remercions également toutes les personnes qui ont répondu à notre enquête et nous ont fourni des documents et des contacts, ainsi que les évaluateurs anonymes de Critique internationale et les coordinateurs du présent dossier thématique pour leurs remarques sur les premières versions de notre article. Notre recherche de terrain a été possible grâce à une bourse accordée par la Commission franco-américaine Fulbright (Programme « Chercheurs », 2010) et elle a bénéficié de l'accueil de l'Institute for Research on Women à Rutgers University (New Jersey). Les entretiens et les matériaux d'archives en anglais sont traduits par nos soins.

11. En anglais «sisterhood». Cette notion fait référence à la solidarité des femmes par-delà les frontières et leurs différences. Pour des recueils de contributions formulées dans cet esprit, voir Robin Morgan (ed.), Sisterhood Is Powerful. An Anthology of Writings from the Women's Liberation Movement, New York, Vintage Books, 1970 (Random House, 1973) ; R. Morgan (ed.), Sisterhood Is Global. The International Women's Movement Anthology, New York, Anchor Press/Doubleday, 1984 (Feminist Press, 1996).

12. En anglais «East/West divide ». Pour une réflexion sur cette thématique et sur le « dépassement »du clivage, voir Mihaela Frunză, Theodora Eliza Văcărescu (ed.), Gender and the (Post) East/West Divide, ClujNapoca, Limes, 2004.

13. Lettre du 29 octobre 1990, signée «In sisterbood, Ann Snitow for the Organizing Committee in Formation » (archives de Ann Snitow).

14. http://www.leftforum.org/about/history. Pour davantage de détails, I. Cîrstocea, « Après la chute du Mur de Berlin : voies et formats du féminisme transnational », cité. 
« Est-Ouest » dans un cadre non officiel eut lieu en juin 1991 à Dubrovnik (en Yougoslavie à l'époque) et réunit une soixantaine de participantes.

\section{Les fondatrices}

Initiatrice et leader symbolique du NEWW, Ann Snitow est professeure de littérature et d'études sur le genre à l'Eugene Lang College de la New School for Social Research (NSSR). Née en 1943 dans une famille éduquée de la classe moyenne et politisée à gauche ${ }^{15}$, elle est titulaire d'un doctorat en littérature de l'Université de Londres obtenu en 1978. Connue depuis les années 1970, dans les milieux états-uniens, pour être une féministe radicale et une militante pacifiste, elle est décrite par ses collègues comme « une organisatrice brillante » et « une centrale féministe à elle toute seule $\gg^{16}$. De fait, elle a participé à la création des programmes d'études sur les femmes et le genre à Rutgers University (New Jersey) et à la NSSR, coédité des anthologies de textes féministes, contribué à de nombreux ouvrages collectifs et publications périodiques et animé pendant plusieurs années la première émission féministe diffusée sur les chaînes de radio américaines (« Womenkind », lancée en 1970 sur WBAI-FM Pacifica Radio New York). Outre sa curiosité pour la vie des femmes à l'époque soviétique, ce qui l'attire, dit-elle, dans les pays d'Europe de l'Est, ce sont les bouleversements politiques, qui sont à ses yeux l'occasion de renouveler la réflexion et l'action forgées dans l'expérience du mouvement américain pour la libération des femmes : « Elles [les femmes est-européennes] allaient connaître le capitalisme et elles pensaient que ce serait formidable ; mais nous, ici, nous savions qu'il allait y avoir des problèmes - le néolibéralisme en train de s'installer à ce moment-là était terrible $\gg^{17} ; \ll$ Nous étions des féministes radicales, nous aurions aimé que le postcommunisme soit une période de renouvellement du militantisme social, un temps de nouvelles opportunités et de changement $\gg^{18}$.

D'autres personnes l'entourent, qui partagent son histoire et/ou ses dispositions politiques : des universitaires spécialistes des études sur les femmes, sur le genre ou l'aire culturelle est-européenne et, plus généralement, des femmes s’intéressant à la région ex-soviétique en raison de leurs parcours individuels et familiaux marqués par l'exil ou par un positionnement politique à gauche. Nanette Funk par exemple

15. Virginia Snitow (1911-2000), sa mère, enseignante et militante, fut membre du Parti communiste pendant les années 1930, puis de l'American Jewish Congress, dont elle organisa et dirigea pendant longtemps la section féminine (http://jwa.org/encyclopedia/article/snitow-virginia).

16. Entretiens avec Nanette Funk (New York, 13 septembre 2010), Joanna Regulska (New Brunswick, 19 octobre 2010), Sonia Jaffe Robbins (New York, 3 octobre 2010). Selon ses propres termes, Ann Snitow est « une féministe qui a eu un emploi à l'Université » (entretien, New York, 2 octobre 2010). Pour une notice biographique, voir Barbara Love, Feminists Who Changed America (1963-1974), Urbana, University of Illinois Press, 2006, p. 433.

17. Entretien, New York, 2 octobre 2010.

18. Entretien, New York, 19 septembre 2010. 
(née en 1942), qui enseignait la philosophie et les études allemandes au Brooklyn College de la City University of New York (CUNY) en 1990 et militait dans divers groupes féministes marxistes, participait aux congrès des universitaires socialistes depuis les années 1970 et avait voyagé en Europe de l'Est pour ses recherches. En entretien, elle mentionne ses contacts en Allemagne de l'Est, en Yougoslavie, en Hongrie et en Russie et évoque les familles de ses grands-parents vivant à Bratislava et à Belgrade. Présente à Berlin au moment de la chute du Mur, elle raconte sa « fascination » devant la «transformation en train de se produire» et trouve « extrêmement passionnante » la possibilité d'y assister, tout en s'interrogeant sur la condition des femmes et les modalités de luttes contre les formes d'injustice de genre en voie d'émerger ${ }^{19}$. Elle décrit son engagement auprès du NEWW comme une expérience intellectuelle et politique stimulante qui méritait vraiment qu'elle «y consacre sa vie ». À un moment où les énergies politiques s'essoufflaient aux États-Unis, s'orienter vers l'Europe de l'Est était également un moyen de rester politiquement active en reconvertissant un habitus militant : « Il ne se passait pas grand-chose en matière de féminisme par ici à la fin des années 1980, le mouvement était entré dans une sorte de mort clinique... $\gg^{20}$.

Sonia Jaffe Robbins (née en 1942) fut l'une des principales animatrices au quotidien du NEWW pendant les premières années. Issue d'une famille qui avait subi le maccarthysme - plusieurs de ses membres étaient engagés politiquement ${ }^{21}$ - et titulaire d'un diplôme universitaire de littérature et de journalisme, elle militait dans le mouvement pour la libération des femmes depuis les années 1970. Lorsque le réseau fut lancé, elle était en train de préparer des concours pour l'obtention d'un poste permanent à New York University. Elle décida alors d'abandonner toute ambition de carrière académique et de se consacrer pleinement au travail militant. En 1993, elle devint la responsable du secrétariat du NEWW et ce jusqu'en 1996. Cette expérience est, selon ses propres termes, « la plus passionnante de [sa] vie »; l'occasion ainsi offerte de rencontrer des femmes est-européennes et de développer des solidarités transatlantiques était un moyen de renouer avec ses racines politiques et sociales. Comme pour Nanette Funk, la mise en place du NEWW a permis, selon Sonia Jaffe Robbins, la renaissance d'une politique féministe : «Il y avait une sorte de reflux du féminisme aux États-Unis, une réaction violente (backlash) des républicains contre le libéralisme, le féminisme, les droits. (...) Dubrovnik nous a apporté un élan immense (...), le NEWW nous a aidées à retrouver notre énergie militante $\gg^{22}$.

19. Entretien, New York, 13 septembre 2010.

20. Ibid. Sur le « recul », voire le « retour de bâton » en matière de droits et de mobilisations des femmes aux États-Unis pendant la décennie 1980, Susan Faludi, Backlash: The Undeclared War against American Women, New York, Crown, 1991 (Three Rivers Press, 2006).

21. Entretien, New York, 3 octobre 2010 ; Sonia Jaffe Robbins, « What Did I Knew and When Did I Knew It? », dans Judy Kaplan, Linn Shapiro (eds), Red-Diapers: Growing-up in the Communist Left, Urbana, University of Illinois Press, 1998, p. 103-109.

22. Entretien, New York, 3 octobre 2010. 
Joanna Regulska (née en 1951) est à l'origine des relations institutionnelles du NEWW avec les promoteurs américains de la démocratie en Europe de l'Est. Géographe de formation, elle est née en Pologne dans une famille d'intellectuels de la classe supérieure. Après avoir quitté son pays au début des années 1980, elle a suivi des études doctorales aux États-Unis et entamé une carrière académique qui comportait, entre autres, la participation au développement des programmes d'études sur les femmes et le genre à Rutgers University. À la fin des années 1980, elle organisa dans cette même institution un programme d'échanges internationaux, qu'elle décrit rétrospectivement comme une forme sui generis de militantisme féministe par-delà les frontières, dont l'objectif était d'impulser et de soutenir le développement des enseignements et des recherches sur les femmes et le genre dans les universités est-européennes ${ }^{23}$. Depuis le début des années 1990 elle a participé à de nombreux projets internationaux orientés vers la réforme de l'administration locale dans son pays d'origine et a été également très active dans la mise en place des études sur le genre en Europe de l'Est et dans l'ex-URSS, notamment en dirigeant quelques-uns des programmes de la Central European University et de l'OSI. Véritable figure de passeur culturel, elle interprète son engagement aux côtés du NEWW et plus généralement ses projets en Pologne et en Europe de l'Est comme un moyen de stabiliser son identité éclatée d'exilée et de la valoriser en tant que ressource pour une politique féministe transnationale. Enfin, sans nécessairement devenir des membres formelles du NEWW, des figures du monde intellectuel et militant de la Côte est, connues pour leur engagement dans les études féministes ou dans la défense - y compris institutionnelle - des droits des femmes, ont fréquenté les réunions et investi dans le réseau soutien symbolique et aide concrète. Ellen Willis, Barbara Ehrenreich, Katta Pollitt, Vera Zolberg, Zillah Eisenstein, Mariam Chamberlain, Marjorie Lightman et Rhonda Copelon ${ }^{24}$ ont ainsi constitué un cercle de sociabilité qui assurait les relations du NEWW avec les milieux aussi bien universitaires que philanthropiques et politiques états-uniens.

Du côté est-européen, le réseau était représenté au moment de son lancement par Slavenka Drakulić (née en 1949)25, alors membre d'un groupe d'intellectuelles féministes constitué à la fin des années 1970 en Yougoslavie ${ }^{26}$. Très active et particulièrement visible dans les cercles internationaux, elle a co-signé une contribution

23. Entretien, New Brunswick, 19 octobre 2010 ; page personnelle de Joanna Regulska sur le site de l'Université Rutgers (http://sciencewomen.rutgers.edu/sites/default/files/resume/128cv.pdf) et entretien biographique recueilli par Slawomira Walczewska dans le cadre du projet « Global Feminisms » de l'Université de Michigan (http://hdl.handle.net/2027.42/55694).

24. Pour des notices biographiques, voir B. Love, Feminists Who Changed America (1963-1974), op. cit..

25. http://slavenkadrakulic.com.

26. Sur son histoire et ses activités, voir Zsofia Lorand, «Feminism as Counterdiscourse in Yugoslavia in Two Different Contexts », mémoire de master, Central European University Budapest, 2007 ; C. Bonfiglioli, «Belgrade 1978. Remembering the Conference "Drugarica Zena. Zensko Pitanje - Novi Pristup?/Comrade Woman. The Women's Question: A New Approach?" 30 Years After », mémoire de master, Utrecht University, 2008. 
dans le recueil Sisterhood Is Global27, a participé à différentes manifestations, dont les Socialist Scholars' Conferences à New York, des académies internationales d'été en sciences sociales, des rencontres littéraires. Interlocutrice privilégiée des féministes nord-américaines s'intéressant à l'Europe de l'Est, elle fut la protagoniste des échanges à l'origine du NEWW. Le carnet d'adresses de femmes vivant dans différents pays ex-socialistes, qu'elle constitua pour documenter un reportage ${ }^{28}$ publié en 1990 dans $M s$. (magazine féministe américain fondé par Gloria Steinem), fut une ressource majeure pour l'organisation de la conférence inaugurale du réseau à Dubrovnik.

L'engagement transatlantique de ces femmes traduit des mécanismes subjectifs et sociaux complexes, qui combinent logiques personnelles et logiques politiques, et correspondent également aux conditions particulières de la fin de la guerre froide. Toutes ont en commun une représentation de la chute du Mur de Berlin faisant de cet événement un moment d'ouverture de nouveaux espaces et de nouvelles possibilités d'action politique féministe. Les Nord-Américaines cherchent, d'une part, à élaborer des idées progressistes pour injecter une pensée féministe à la base même des nouvelles démocraties, d'autre part, à alimenter leurs cursus académiques de nouveaux projets de recherche, contacts et publications. Par ailleurs, leur investissement politique orienté vers l'Europe de l'Est se nourrit également d'une propension à renouer avec des passés familiaux ${ }^{29}$. Enfin, échanger avec les femmes des pays ex-socialistes est l'occasion de confronter des idées radicales à l'écho de l'expérience vécue du socialisme : formées dans un contexte libéral, les féministes états-uniennes considèrent les politiques socialistes comme une référence positive et comme un modèle d'émancipation féminine précieux. Or certains témoignages personnels ainsi qu'une importante littérature consacrée pendant les années 1990 au «malentendu Est-Ouest » montrent que la confrontation des représentations du socialisme portées respectivement par les insiders est-européennes et les outsiders occidentales a pu se révéler difficile et douloureuse ${ }^{30}$. D'ailleurs, ce n'est pas un hasard si le NEWW s'appuya d'abord en Europe de l'Est sur un partenariat avec la Yougoslavie où eut lieu justement son lancement. Dans ce pays « ouvert » et considéré par ses ressortissantes comme « en dehors de la division culturelle et politique entre Est et Ouest $\gg^{31}$, les femmes se mobilisaient indépendamment des cadres politiques étatiques : elles écrivaient et diffusaient

27. Slavenka Drakulić, Rada Iveković, « Neofeminism and Its Six Mortal Sins », dans R. Morgan (ed.), Sisterhood Is Global. The International Women's Movement Anthology, op. cit., p. 737-741.

28. S. Drakulić, « In Their Words. Women of Eastern Europe », Ms., juillet-août 1990, p. 36-47.

29. Nos interlocutrices emploient le terme « résilience » pour désigner le rapport émotionnel avec l'Europe de l'Est, leur mémoire familiale et leur engagement politique transnational pendant les années 1990.

30. Nanette Funk, « Fifteen Years of the East-West Women's Dialogue », dans Janet Elise Johnson, Jean C. Robinson (eds), Living Gender after Communism, Bloomington, Indianapolis, Indiana University Press, 2007, p. 203-226.

31. S. Drakulić, «In Their Words. Women of Eastern Europe », art. cité, p. 46. La position « non alignée » du pays pendant la guerre froide continue d'être valorisée en termes similaires aujourd'hui encore (entretiens personnels, Paris, 2010, Belgrade et Dubrovnik, 2014). 
des productions critiques, organisaient des groupes de soutien et étaient reliées à l'international. Le réseau transatlantique se mit donc en place pour valoriser ces expériences, les prolonger et les élargir.

Déterminée essentiellement par une logique d'extension et de changement d'échelle des solidarités militantes, l'action des féministes s'inscrit également dans le cadre plus général de la prolifération, pendant les années 1990, des entreprises vouées à l'exportation de la société civile, de la politique des droits et des normes du marché dans les pays ex-socialistes. Des liens formels et informels unissaient entre elles ces initiatives dans lesquelles s'enchevêtraient les mobilisations féministes transatlantiques ; les promotrices du genre dans les espaces nationaux est-européens furent par ailleurs souvent des figures d'« entrepreneures de démocratie » et des interlocutrices privilégiées des programmes internationaux.

\section{Le lancement du NEWW et ses suites}

Après une série de rencontres et des échanges soutenus de correspondances au cours de l'été 1990, une invitation fut adressée par les fondatrices du NEWW à quelques dizaines de femmes est-européennes : «Nous sommes un groupe de travail espérant pouvoir devenir un réseau de femmes féministes ; nous sommes toutes écrivaines, professeures, militantes féministes. (...) Nous apprenons avec inquiétude que les femmes de l'Est sont confrontées au risque de perdre leurs droits (...). Notre objectif consiste d'abord à faire naître un dialogue est-européen, à travers les frontières nationales, ensuite à transmettre notre expérience quant au développement d'un mouvement de libération des femmes, à partager nos succès mais aussi nos échecs dans le combat pour les droits des femmes dans une économie de marché $»^{32}$.

Les organisatrices portèrent leur choix sur la ville de Dubrovnik, réputée, certes, pour son cadre naturel et son histoire mais, surtout, pour les activités de l'InterUniversity Center ${ }^{33}$, hôte d'écoles d'été et de conférences internationales ${ }^{34}$. Les fonds recueillis par le noyau fondateur du NEWW permirent de couvrir les frais de déplacement et de séjour de la soixantaine de femmes qui s'y réunirent du 7 au 9 juin 1991 : 19 Nord-Américaines, 21 Yougoslaves, 9 Polonaises, 5 Tchèques, 5 Allemandes, 4 Bulgares, une Roumaine, une Grecque et une Ukrainienne ;

\footnotetext{
32. Lettre du 29 octobre 1990, signée «In sisterhood, Ann Snitow for the Organizing Committee in Formation » (archives de Ann Snitow).

33. http://www.iuc.hr. Consortium académique mis en place en 1972 pour faciliter les contacts intellectuels entre les deux blocs idéologiques, l'Inter-University Center réunit plusieurs dizaines d'universités de tous les continents. Il a bénéficié du support des Nations unies et, depuis la fin des années 1980, de la Fondation Soros. 34. Quelques-unes d'entre elles touchaient à des thématiques féministes - de la sociologie du travail des femmes à la philosophie et à la critique littéraire féministes - et offraient une tribune à des débats théoriques et à des figures intellectuelles d'avant-garde. Nanette Funk y a participé et a rencontré de futures amies est-européennes (entretien, New York, 13 septembre 2010). Reprises après les guerres des années 1990, ces rencontres continuent aujourd'hui de fonctionner comme un cadre de réflexion féministe transatlantique.
} 
certaines d'entre elles seront longtemps présentes sur la scène politique et intellectuelle est-européenne et internationale des droits de l'homme et des droits des femmes ${ }^{35}$. Parmi les participantes, il y en avait quelques-unes qui ne connaissaient alors rien au féminisme, d'autres quittaient leurs pays pour la première fois, certaines enfin vinrent accompagnées de membres de leurs familles.

Des sessions thématiques et des ateliers figuraient au programme de la manifestation, ainsi que des groupes de prise de parole. Ann Snitow souligna dans son allocution d'ouverture l'importance de la solidarité féministe et du militantisme internationaux, capables, d'après elle, d'inspirer et de soutenir l'émergence de groupes militants au niveau national. Autour du slogan « La démocratie sans les femmes n'est pas une (vraie) démocratie », Slavenka Drakulić fit également une intervention en réunion plénière. Centré sur la dégradation de la condition des femmes dans les pays postsocialistes, son exposé citait un rapport des Nations unies dénonçant la baisse de la représentation politique, la ségrégation sexuée du monde du travail, la montée de la pauvreté, la prolifération de la pornographie et de la prostitution, ainsi que les menaces à l'encontre de la liberté des droits reproductifs dans ces pays. Toutes deux insistèrent sur le potentiel émancipateur de la « sororité », entendue comme la condition commune des femmes par-delà leurs différences, ainsi que sur la nécessité de bâtir des solidarités à travers les frontières afin de faire entendre les revendications féministes et de les faire peser dans le débat politique.

Parmi les thèmes abordés dans les ateliers, mentionnons à titre d'exemple : femmes et famille, femmes et travail, sexualité et santé de la reproduction, participation et représentation politique des femmes, modèles d'organisation féministe et stratégies pour mettre en place des recherches et des enseignements sur le genre. Les participantes qui avaient préparé et fait circuler leurs contributions à l'avance étant rares, le programme de la conférence fut finalement très souple, laissa du temps pour de longs échanges informels et connut même des retournements qui pouvaient déstabiliser les organisatrices. Ainsi, l'atelier sur le travail, qui devait être animé par une dirigeante syndicale polonaise, n'eut finalement pas lieu, faute de volontaires ${ }^{36}$. Dans les échanges féministes transatlantiques des années 1990, les représentations différentes du travail féminin deviendront une thématique centrale du «malentendu Est-Ouest » : face aux Européennes de l'Est qui critiqueront l'obligation de travailler imposée par les régimes socialistes, les Américaines insisteront sur l'importance de l'indépendance économique des femmes. De même, le groupe de prise de parole (consciousness raising group, élément classique du répertoire d'action des féministes américaines depuis les années 1970), vivement recommandé comme outil permettant de se connaître, de

35. Par exemple Malgorzata Tarasiewicz, directrice de NEWW-Polska, ou Sonja Licht, présidente de l'Open Society Foundation Serbie de 1991 à 2003.

36. Entretien avec Sonia Jaffe Robbins, New York, 3 octobre 2010. 
formuler des problèmes communs et d'ouvrir la voie à la construction politique de la solidarité des femmes, apparut choquant pour certaines participantes esteuropéennes qui le trouvèrent intrusif et n'acceptèrent pas l'idée d'aborder leurs expériences intimes devant des inconnues ${ }^{37}$. Cet exemple renvoie lui aussi à la « division » : différentes définitions culturelles de l'intimité aussi bien qu'une réaction défensive pouvant être attribuée à l'expérience du contrôle politique exercé par les régimes communistes sur la vie privée des citoyens firent que les Européennes de l'Est se méfiaient du slogan féministe occidental « le personnel est politique ». Enfin, accoutumées à des réunions politiques formalisées, voire rigidement organisées, quelques-unes d'entre elles raillèrent les conversations décontractées sur des expériences quotidiennes des femmes, les trouvant «pas assez sérieuses », voire dépourvues de tout intérêt. De tels incidents annoncent bien ce qui allait être vécu et amplement analysé par la suite en termes de « choc culturel ». Durant les entretiens, nos interlocutrices ont évoqué leur étonnement d'alors face à cette altérité insoupçonnée, révélée par la rencontre transatlantique, ainsi que la difficulté pratique et symbolique à proclamer l'identité collective de femmes qui n'avaient pas les mêmes expériences politiques et sociales.

La rencontre s'acheva par une discussion générale sur les suites possibles à donner aux échanges internationaux. Il est utile d'en détailler ici le contenu si l'on veut restituer la vision et l'énergie du groupe, à la fois source et référence de ses activités pendant les années qui ont suivi. Il fut ainsi question de créer un groupe de pression auprès des Nations unies; de constituer et d'entretenir une base de données réunissant les coordonnées des personnes ressources pour le féminisme en Europe de l'Est; d'établir l'infrastructure nécessaire pour la communication internationale, l'échange d'informations et le travail sur des projets communs (la question d'un réseau informatique fut évoquée); de publier un bulletin périodique d'information ainsi que des rapports par pays sur toutes les questions relatives aux droits des femmes; d'organiser une chaîne téléphonique internationale ; de diffuser en direction des publics est-européens des classiques de la littérature féministe américaine ; de mettre en place des programmes d'échanges académiques, bourses et stages d'apprentissage dans les institutions états-uniennes et internationales ; d'aider à la fondation en Europe de l'Est de centres d'études, programmes et associations féministes; de lancer à cette fin des collaborations avec les agences internationales déjà présentes dans la région ${ }^{38}$. Une déclaration commune adoptée à la fin de la réunion et diffusée largement grâce aux journalistes présents sur place résumait tous ces éléments et soulignait l'ambition du NEWW de contribuer au « développement d'un mouvement populaire des femmes en Europe centrale et orientale ».

37. Entretiens avec Nanette Funk, New York, 13 septembre 2010, et Sonia Jaffe Robbins, New York, 3 octobre 2010 .

38. Notes de Ann Snitow au Congrès de Dubrovnik. 
Après la rencontre de Dubrovnik, le réseau fonctionna dans un premier temps de manière informelle. Un certain nombre de participantes, pouvant aller jusqu'à 50, se rencontraient régulièrement dans l'appartement de Ann Snitow et restaient en contact via une chaîne téléphonique. Un groupe de réflexion fut mis en place à New York University, qui devint par la suite un séminaire régulier, encore actif aujourd'hui, centré sur la présentation et la discussion de travaux portant sur les pays ex-socialistes ${ }^{39}$. Des annuaires réunissant les contacts des membres et sympathisantes, ainsi qu'un bulletin périodique furent également créés, le dernier ayant pour vocation de collecter et de diffuser des informations sur les conférences et les publications, les projets, les fonds disponibles pour la recherche et les nouvelles initiatives féministes dans les pays d'Europe de l'Est. Des évolutions sociales et politiques susceptibles de faire l'objet d'une réflexion et d'une mobilisation féministes furent abordées en présence de juristes et d'expertes américaines : par exemple, des projets de lois entravant les droits des femmes comme la loi sur l'avortement en Hongrie ou la nouvelle Constitution en Russie ; la question des viols de guerre en Yougoslavie, abordée aussi dans le cadre de la campagne «Women's Rights Are Human Rights » lancée par le Centre for Global Women's Leadership (Rutgers University). Le groupe informel se faisant assez rapidement déborder par l'ampleur des échanges ${ }^{40}$, la question de la normalisation fut soulevée et le NEWW en vint à adopter le format institutionnel d'une ONG, se dotant ainsi d'une identité lisible sur le marché des projets associatifs. Des services professionnels de consultance furent sollicités et le réseau embaucha des personnes dotées d'une expérience managériale, capables de travailler avec des appels de fonds et des budgets comptables.

La solidarité féministe transatlantique visait presque exclusivement la « région » ex-socialiste et ignorait les liens avec les autres femmes européennes, car le NEWW mettait en avant dans la définition de son identité militante le clivage « Est-Ouest », solidement ancré dans les représentations de ses créatrices étatsuniennes. Certes, les contacts et les collaborations avec des Européennes de l'Ouest n'étaient pas exclus, mais ils se limitaient à des échanges fondés sur le partage d'un intérêt pour l'« Est » et les «femmes de l'Est ». Si les groupes féministes mobilisés en Europe de l'Ouest au tournant des années 1990 ne privilégiaient pas, à quelques exceptions près, la pratique transnationale ${ }^{41}$, les Nord-Américaines,

\footnotetext{
39. http://gendertransformationeurope.wordpress.com.

40. Le secrétariat du réseau recevait 100 messages par jour en 1995, les documents internes font état de 2000 membres et sympathisantes en 1998.

41. Créé vers le milieu des années 1980, le Forum européen des féministes socialistes invitait des ressortissantes des pays socialistes (Fiona Williams, « A Report on the 5th Annual Conference of the European Forum of Socialist-Feminists, Gothenburg, Sweden, November 24-26, 1989 », Critical Social Policy, 10, 1990, p. 96102 ; Cynthia Cockburn, « The European Forum of Socialist Feminists: Talking on the Volcano », Women's Studies International Forum, 15 (1), 1992, p. 53-56). Le Lobby européen des femmes se met en place en 1991 ; au tournant des années 1990, le Conseil de l'Europe commence à soutenir la mise en réseau des programmes académiques nationaux de women's studies.
} 
elles, bénéficiaient d'importantes ressources précisément orientées vers une telle finalité. Ces ressources provenaient de la conversion des programmes développés dans le cadre de la guerre froide en programmes d'assistance à la démocratisation ou d'étude des processus de transition ${ }^{42}$, dotés de lignes de financement dédiées aux femmes des « pays de l'Est », à leurs projets associatifs et à leurs mobilisations pour la défense de leurs droits.

C'est dans ce contexte d'extension des possibilités de financement et des voies de carrière que s'inscrivent la formalisation et la professionnalisation du NEWW. Le processus de normalisation, dont nous ne pouvons détailler ici les ressorts ${ }^{43}$, doit être compris à la lumière d'un contexte sociopolitique particulier, notamment la prolifération des supports accessibles sur le marché états-unien et international de l'assistance à la démocratisation. Cette logique se traduisit par la cooptation dans le réseau d'expertes dotées de compétences sur l'« Est $\gg^{44}$, c'est-à-dire spécialistes, par leurs études ou par une expérience professionnelle antérieure, de la région soviétique et des échanges internationaux. Le savoir-faire, les contacts, mais aussi les dispositions pratiques et idéologiques constituées avant leur arrivée au NEWW furent investis dans la formalisation du réseau. Les projets institutionnels démarrèrent en 1994 grâce à leur concours et bénéficièrent du soutien financier de fondations et de programmes gouvernementaux américains et internationaux ${ }^{45}$.

Outre le processus de stabilisation sous forme d'ONG, le NEWW a connu depuis sa fondation plusieurs moments (parfois conflictuels) de redéfinition de ses objectifs, de son identité et de sa stratégie, tandis que le périmètre des pays visés par ses programmes évoluait au gré des mutations géopolitiques ${ }^{46}$. Doté d'une branche polonaise depuis la fin des années $1990^{47}$, il est aujourd'hui basé administrativement à Gdansk et reconnu dans le monde comme l'une des plus actives et des plus visibles institutions régionales de défense des droits des femmes. Le groupe des fondatrices nord-américaines poursuit ses activités de réseautage et d'échanges informels tout en cultivant une dimension mémorielle autour du séminaire new-yorkais et de leurs activités d'enseignement, mais aussi de leurs écrits académiques et littéraires, qui restituent parfois des éléments de l'histoire du NEWW. Enfin, les pionnières est-européennes du genre retiennent les activités

\footnotetext{
42. Nicolas Guilhot, The Democracy Makers. Human Rights and International Order, New York, Columbia University Press, 2005.

43. I. Cîrstocea, « "Comment être démocratique tout en étant réaliste ?”. Le militantisme féministe à l'épreuve de la transnationalisation (NEWW, 1990-2000) », communication au Congrès de l'Association belge francophone de science politique, Liège, 10 et 11 avril 2014.

44. Par exemple Shana Penn, directrice exécutive du NEWW à partir de l'automne 1993, ou Melissa L. Stone, secrétaire du bureau directeur à partir d'avril 1994.

45. I. Cîrstocea, « Après la chute du Mur de Berlin : voies et formats du féminisme transnational », cité.

46. Par exemple les attentats du 11 septembre 2001 et l'adhésion à l'Union européenne de certains pays ex-socialistes en 2004 et 2007, suivis par la redéfinition de l'aire d'intervention de l'aide internationale et la reconfiguration du spectre de ses bénéficiaires.

47. http://www.neww.org.pl.
} 
du réseau comme une référence importante pour l'émergence de la réflexion théorique et pour le développement de la pratique et de l'expertise féministes dans l'espace ex-socialiste ${ }^{48}$.

\section{Les ressources idéologiques du féminisme international : " sororité globale » et " malentendu Est-Ouest »}

Au début des années 1990, lors du lancement du NEWW, la « sororité » était une référence bien établie dans la pratique ainsi que dans le répertoire idéologique des groupes militants occidentaux actifs dans les mouvements pour la libération des femmes. Les animatrices américaines du réseau proviennent de ces groupes et réactualisent leurs dispositions militantes en inventant une forme de solidarité qui dépasse les cadres de leur activisme politique d'avant la fin de la guerre froide. Elles font revivre la référence à cette solidarité féministe en dépit des critiques - qu'elles n'ignorent pas - des courants de pensée non hégémoniques qui, au cours de la décennie 1980, se sont réclamés du Black Feminism et du féminisme postcolonial pour dénoncer l'homogénéité du sujet « femmes » et la solidarité féministe universelle, et faire reconnaître les différences entre les femmes ainsi que les formes de domination complexes, au croisement de différents rapports sociaux (classe, race, positionnement géopolitique, etc. ${ }^{49}$.

Si elles incarnent effectivement la catégorie hégémonique des « féministes occidentales »- elles sont blanches, éduquées, disposent de ressources économiques relativement importantes et sont citoyennes d'un État superpuissant -, les militantes du NEWW basées aux États-Unis ne composent pas elles-mêmes un groupe homogène, et leur pratique de la solidarité transnationale n'est pas aveugle aux inégalités entre les femmes. Lorsqu'elles lancent leur appel au dialogue international - donnant ainsi une nouvelle impulsion à leurs propres carrières (militantes et académiques) - elles s'appuient sur un répertoire « historique » qui renvoie non seulement à leurs propres parcours - personnels, familiaux et politiques - dans l'espace national états-unien, mais aussi à l'histoire des mouvements de libération des femmes au sens large, dont on sait que la pratique de la coopération internationale a été à la fois une ressource stratégique, un outil de combat politique et un enjeu proprement $\mathrm{dit}^{50}$. Dans le contexte (géo)politique de l'après-guerre froide, imaginé comme un moment propice au renouvellement de l'internationalisme, elles procèdent à un changement d'échelle de leur action et déplacent leur engagement du niveau national au niveau transnational.

48. Pour un témoignage roumain, Laura Grünberg, Bi(ONG)rafie. AnA - istoria trăită a unui ONG de femei (Bi(ONG)graphie. AnA - histoire vécue d'une ONG de défense des droits des femmes), Iaşi, Polirom, 2008.

49. Pour une collection de textes inspirés de cette réflexion, Christine Verschuur (dir.), « Genre, postcolonialisme et diversité des mouvements des femmes », Cahiers Genre et Développement, dossier thématique, 7, 2010.

50. Anne Taylor Allen et al. (eds), «International Feminisms », Women's History Review, dossier thématique, $19,4,2010$. 
Pour résoudre la question cruciale de la définition du féminisme - en tout cas d'une définition a minima qui serait la mieux à même d'orienter concrètement la pratique de la solidarité internationale -, les animatrices du NEWW s'attachent à un principe à la fois théorique et opérationnel, qui consiste à considérer les inégalités de genre comme des invariants anthropologiques différemment actualisés selon le contexte socio-historique et politique. Dès lors, l'objectif du dialogue transatlantique n'est pas de trouver et d'imposer des solutions universelles pour les problèmes des femmes, mais de cultiver des dispositions critiques et d'identifier, dans des lieux spécifiques, les questions qui seraient à politiser dans une perspective féministe. Pour citer Ann Snitow, il s'agit de « repérer des points d'entrée pertinents pour la pensée féministe », voire de « comprendre ce que le féminisme a besoin d'être dans une situation donnée $\gg^{51}$. Les Américaines se considèrent elles-mêmes comme des « accoucheuses » (facilitators, enablers) de nouveaux féminismes et elles regardent leur activisme en direction des pays ex-socialistes comme une initiative censée contribuer à l'autonomisation (empowerment) des femmes de l'Est ${ }^{52}$. Leur pratique militante comme leur réflexion permanente sur cette pratique montrent combien elles ont conscience de leur position privilégiée : enable, empower ne sont pas des mots neutres, ils désignent une nette inégalité de ressources. Les écarts économiques et technologiques qui font réellement obstacle à la pratique de la solidarité transatlantique ne sont ni ignorés ni passés sous silence dans la pratique du NEWW. Le choix pragmatique de ces militantes consiste à répondre au rapport inégalitaire en inventant une forme sui generis de « politique transversale », une utopie féministe fondée sur des interactions non hiérarchiques et une organisation transparente, s'appuyant sur l'usage engagé des nouvelles technologies ${ }^{53}$ et sur le soutien collégial de la profession académique et de la société civile professionnalisée et internationalisée.

Le réseau traduit l'insertion des Européennes de l'Est (de certaines d'entre elles du moins) dans les arènes du féminisme globalisé et impulse l'émergence d'une « voix différente » dans le chœur féministe. En effet, dans le cadre des échanges transatlantiques et grâce à eux, la catégorie identitaire « femmes esteuropéennes » a pu être investie politiquement et utilisée comme support d'affirmation de nouvelles actrices dans l'espace international de l'expertise sur le genre. De nouvelles figures de féministes ont connu à ce moment-là une consécration

51. Entretien avec Ann Snitow, New York, 2 octobre 2010 ; voir aussi A. Snitow, « Feminist Future in the Former East Bloc », dans Marina Blagojević et al. (eds), What Can We Do For Ourselves? East-European Feminist Conference, Belgrade, Center for Women's Studies, Research and Communication, 1995, p. 141-155 ; A. Snitow, «Cautionary Tales », dans Jasmina Lukić et al. (eds), Women and Citizenship in Central and Eastern Europe, Aldershot, Ashgate, 2006, p. 287-297.

52. Entretien avec Debra Shultz, membre du NEWW et co-responsable d'un programme « femmes » à l'OSI (New York, 29 septembre 2010).

53. Pour une réflexion de synthèse sur le potentiel et les limites du militantisme transnational «à distance », voir Elisabet Jay Fridman, « The Reality of Virtual Reality: The Internet and Gender Equality Advocacy in Latin America », Latin American Politics \& Society, 47 (3), 2005, p. 1-34. 
internationale par leurs participations à des conférences, leurs publications, leurs activités de recherche et d'enseignement menées dans des cadres transnationaux, leurs missions de consultance et leurs emplois dans d'importantes organisations internationales. Un exemple parmi d'autres, et que nous avons présenté plus en détail ailleurs, est celui de Anastasia Posadskaya, économiste russe, directrice du Centre d'études sur le genre à Moscou, et pressentie un moment pour être la leader est-européenne du NEWW. Après avoir participé à différentes activités du réseau, elle intégra l'OSI, dont elle a codirigea le programme de promotion des droits des femmes pendant plusieurs années, puis travailla pour différentes structures internationales, dont l'ONU ${ }^{54}$.

Paradoxalement, tout en aspirant à créer des liens par-delà les frontières et en le faisant concrètement à travers de nombreux projets régionaux et transatlantiques, la solidarité internationale au sein du NEWW est aussi productrice de différences. On peut donc la considérer comme une pratique performative, qui opère avec la catégorie « femmes est-européennes » tout en l'instituant. Indépendamment de sa définition géographique, historique et culturelle - qui n'est pas stable -, la notion $\mathrm{d}^{\prime}$ « Europe de l'Est $\gg^{55}$ a aussi une dimension idéologique attachée au répertoire de représentations propres à la guerre froide. Dans le cadre que nous avons analysé ici, les « femmes est-européennes » sont les femmes socialisées à l'époque du régime soviétique et, à ce titre, projetées symboliquement comme des figures de l'altérité. Au cours des années 1990, à mesure que se développaient les échanges internationaux, elles ont appris à revendiquer collectivement cette altérité tout en se faisant une place sur la carte du féminisme international. Pendant un certain temps, les activités développées autour du NEWW ont contribué à faire perdurer le « Deuxième Monde » après la chute effective du Mur de Berlin et ont solidifié l'expérience du socialisme d'État en marqueur d'identité politique au sein de l'« espace (inter/transnational) de la cause des femmes $»^{56}$.

Des schémas de représentation du monde et des dispositions militantes constituées par la socialisation dans un monde bipolaire ont orienté l'activité des groupes féministes transnationaux pendant la décennie 1990. En ce sens, le NEWW

54. I. Cîrstocea, « Se faire une voix : logiques internationales du féminisme est-européen (1990-2000) 》, communication au Quatrième Congrès international du réseau francophone des Associations de science politique, Bruxelles, 20-22 avril 2011.

55. Larry Wolff, Inventing Eastern Europe: The Map of Civilization on the Mind of the Enlightenment, Stanford, Stanford University Press, 1994. Sur les représentations orientalistes de l'Europe de l'Est, Maria Todorova, Imaginaire des Balkans, Paris, Éditions de l'EHESS, 2011 (Oxford University Press, 1997).

56. Pour la notion d'« espace de la cause des femmes » que nous adaptons ici à une échelle transnationale, voir L. Bereni, « De la cause à la loi. Les mobilisations pour la parité politique en France (1992-2000) », thèse de doctorat de l'Université Paris 1 Panthéon - Sorbonne, 2007 ; L. Bereni, « La transnationalisation des mouvements des femmes contemporains. Quelques enjeux d'un champ de recherche », introduction au séminaire «Genre, féminismes et mobilisations collectives », EHESS, CMH-ETT, EFiGiES, 2008-2009 (http://www.melissa.ens-cachan.fr/IMG/pdf/La_transnationalisation_des_mouvements_des_femmes__21nov2008-2.pdf). 
incarne plusieurs passés « qui ne passent pas » et plusieurs histoires entrecroisées : histoire géopolitique du $\mathrm{XX}^{\mathrm{e}}$ siècle, histoire sociale et intellectuelle du féminisme états-unien et du féminisme occidental plus largement, différentes histoires familiales et personnelles marquées par l'exil et le déracinement. L'étude de ce réseau jette une lumière nouvelle sur les entreprises de démocratisation des pays ex-socialistes et permet de comprendre l'importation du répertoire du genre comme innovation institutionnelle, intellectuelle et politique au carrefour de dynamiques nationales, régionales et globales. Enfin, les activités et l'évolution du NEWW pendant ses premières années d'existence illustrent les modalités de déploiement des divisions idéologiques propres à la guerre froide par-delà les cadres chronologiques communément acceptés pour en marquer la fin. Ainsi l'ouverture de nouvelles possibilités de circulation internationale ne change-t-elle spontanément ni les reflexes des acteurs sociaux ni les cadres de représentation définissant leurs pratiques.

loana Cîrstocea est sociologue, chargée de recherche au CNRS, membre de I'UMR 7363 SAGE (Sociétés, Acteurs, Gouvernement en Europe) de I'Université de Strasbourg. Ses recherches portent actuellement sur les transformations des espaces intellectuels et la sociogenèse des études sur le genre en contexte postsocialiste ainsi que sur la production et les circulations internationales de l'expertise féministe. Elle a publié notamment Faire et vivre le postcommunisme. Les femmes roumaines face à la " transition » (Bruxelles, Éditions de I'Université de Bruxelles, 2006); " Éléments pour une sociologie des études féministes en Europe centrale et orientale ", International Review of Sociology/Revue internationale de sociologie (20 (2), 2010, p. 321-346) ; "Les restructurations de l'enseignement supérieur en Roumanie après 1990 : apprentissage international de la gestion, professionnalisation de l'expertise et politisation de l'enjeu universitaire ", Revue d'études comparatives EstOuest (45 (1), 2014, p. 125-163).

loana.Cirstocea@misha.fr 